\title{
Sublattice Identification in Scanning Force Microscopy on Alkali Halide Surfaces
}

\author{
R. Hoffmann, ${ }^{1,2, *}$ L. N. Kantorovich, ${ }^{3}$ A. Baratoff, ${ }^{1}$ H. J. Hug, ${ }^{1,4}$ and H.-J. Güntherodt ${ }^{1}$ \\ ${ }^{1}$ National Center of Competence in Research on Nanoscale Science, Institute of Physics, University of Basel, \\ Klingelbergstrasse 82, CH-4056 Basel, Switzerland \\ ${ }^{2}$ Physikalisches Institut, Universität Karlsruhe, 76128 Karlsruhe, Germany \\ ${ }^{3}$ Department of Physics, School of Physical Sciences and Engineering, King's College London, \\ London WC2R $2 L S$, United Kingdom \\ ${ }^{4}$ Swiss Federal Laboratory for Materials Testing and Research, CH-8600 Dübendorf, Switzerland
} (Received 17 March 2003; published 9 April 2004)

\begin{abstract}
We propose and apply to the $\mathrm{KBr}(001)$ surface a new procedure for species recognition in scanning force microscopy (SFM) of ionic crystal surfaces which show a high symmetry of the charge arrangement. The method is based on a comparison between atomistic simulations and site-specific frequency versus distance measurements. First, by taking the difference of force-distance curves extracted at a few judiciously chosen surface sites we eliminate site-independent long-range forces. The obtained short-range force differences are then compared with calculated ones assuming plausible tip apex models. This procedure allows for the first time identification of the tip apex polarity and of the positive and negative sublattices in SFM images of the (001) cleavage surface of an ionic crystal with the rock salt structure.
\end{abstract}

Since the first reports of true atomic resolution obtained on a $\mathrm{Si}(111) 7 \times 7$ surface with a scanning force microscope (SFM) in a novel dynamic near contact mode [1], this technique has developed into a powerful tool for surface studies of insulators [2]. In particular, alkali halide (001) and alkali earth halide (111) surfaces have been extensively studied because these insulator surfaces are easily accessible to both calculations and experiment. From a theoretical point of view, atomistic simulations based on semiempirical potentials taking the polarization and relaxation of the ions into account have succeeded in giving a detailed understanding of the interaction of these surfaces with plausible models of the probing tip [3]. From an experimental point of view, these surfaces are easy to prepare, and atomic resolution is readily achieved [4-7]. Encouraging agreement between calculations and experiments has been obtained [3,6,7]. In contrast to surfaces of covalent materials such as $\mathrm{Si}(111) 7 \times 7$ on which adatoms with dangling bonds are imaged, on ionic compounds one type of surface ion is imaged as bright, the other as dark, depending on the sign of the electrostatic potential at the tip apex [3,7]. On the $\mathrm{CaF}_{2}(111)$ surface, the sign of the potential could be identified by analyzing the asymmetry of the observed contrast [7]. This was possible because the unit cell contains one $\mathrm{Ca}^{2+}$ ion and two inequivalent $\mathrm{F}^{-}$ions. On the (001) surfaces of ionic crystals with the rocksalt structure, sublattice identification is difficult because the arrangement of the ions is highly symmetric. These surfaces which are commonly obtained upon cleavage or film growth of most group I and II metal halides and oxides, are currently the subject of extensive fundamental, as well as applied studies concerning characteristic point defects $[4-6,8,9]$, adsorbed molecules [10], and controlled deposition of nanopar- ticles [11]. It is essential to know which sublattice is imaged as bright if defects or nanostructures on the surface are to be identified. In this Letter we show how a comparison of measured and calculated forces as a function of tip-sample distance at specific sites can be used for sublattice identification.

Recently, the force on the tip as a function of displacement perpendicular to the sample has been determined above specific atomic sites on the $\operatorname{Si}(111) 7 \times 7$ [12], the $\mathrm{KBr}(001)$ [13], and the $\mathrm{NiO}(001)$ [14] surfaces. This type of measurement is possible thanks to a unique, largely drift-free low-temperature instrument [15]. The measured frequency shift can be converted to tip-sample force; in the case of silicon even the short-range force due to the interaction of a single Si atom at the tip apex with a Si adatom on the surface could be extracted [12]. One problem is the reliable separation of long-range and short-range contributions to the net force since at close tip-sample distances both forces contribute. Above the corner hole on the $\mathrm{Si}(111) 7 \times 7$ surface, the long-range force contributes almost exclusively because all reactive adatoms on the surface are several angströms away. Above Si adatoms the short-range contribution to the force could be revealed by subtracting that long-range contribution. However, on most other samples, in particular, rocksaltlike (001) surfaces, no features like corner holes exist, while short-range forces are comparatively weaker. In this Letter we focus on the differences between forces obtained at selected sites to eliminate the siteindependent long-range force. The force differences measured on the $\mathrm{KBr}(001)$ surface are compared to the corresponding differences obtained from atomistic simulations. The polarity of the tip apex can thus be determined, leading to identification of the surface species 
even in this difficult case where their ionic radii are nearly the same.

The code SCIFI [16] used in our simulations computes forces acting between a system of conducting objects and ions so as to model force microscopy on ionic crystals. Such simulations have previously been performed for $\mathrm{LiF}(001), \mathrm{NaCl}(001)[6,8]$, and $\mathrm{CaF}_{2}(111)$ [7] assuming a cubic $\mathrm{MgO}$ cluster at the tip apex. This cluster supposedly represents a sharp oxidized Si tip because the electrostatic potential around a corner $\mathrm{O}^{2-}$ ion is like that around an oxygen atom bound to the apex of a Si pyramid [8]. For reasons discussed below we have performed calculations for a cubic cluster of the same material as the sample.

The short-range force is computed from a sum of pairwise Buckingham and Coulomb potentials acting between ions treated atomistically. Ions are treated as coupled, oppositely charged cores and shells in order to describe their polarizabilities, with parameters taken from Ref. [17]. The sample was represented by a slab of six layers containing $10 \times 10$ ions; those in the bottom layer and on the sides were kept fixed. The atomistic part of the tip was represented by a cubic $(\mathrm{KBr})_{32}$ cluster oriented such that one $\langle 111\rangle$ direction was perpendicular to the sample. Two such tips were considered: one with a $\mathrm{K}^{+}$ion, and another one with a $\mathrm{Br}^{-}$ion at the apex facing the sample. Ions in the top half of the cluster were kept fixed. The tip was placed above the three positions shown in Fig, 1(a). For each position, the force on the tip was calculated at closely spaced increments towards the sample. At each position, the shell and core coordinates were allowed to relax until mechanical equilibrium was reached; the forces on the fixed tip atoms were then summed. Because sample and tip are charge neutral, the resultant force is short ranged.

The distance dependence of that force calculated for the $\mathrm{K}^{+}$- and the $\mathrm{Br}^{-}$-terminated tips is shown in Fig. 1 for three lateral positions: (i) above a $\mathrm{K}^{+}$ion, (ii) above a $\mathrm{Br}^{-}$ ion, and (iii) above a bridge position midway between two neighboring $\mathrm{K}^{+}$ions and two neighboring $\mathrm{Br}^{-}$ions. The distance is defined as that between the tip apex ion and the top layer of the sample in the absence of relaxation. Shortrange forces are usually first attractive upon approach, reach a minimum, and then become increasingly repulsive. This behavior is obtained above the surface ioll charged oppositely to the tip apex ion. It is also obtained at the bridge position for either tip termination. On an ionic crystal surface, however, a weak short-range repulsive force can first appear upon approach when the ion at the tip apex faces an ion of the same charge on the sample surface before attraction to further neighbors and, eventually, repulsion becomes dominant.

The forces calculated above the bridge position and above the oppositely charged ion differ substantially for the two tip terminations. For the $\mathrm{K}^{+}$-terminated tip, the minimum of the curve obtained at the bridge position is
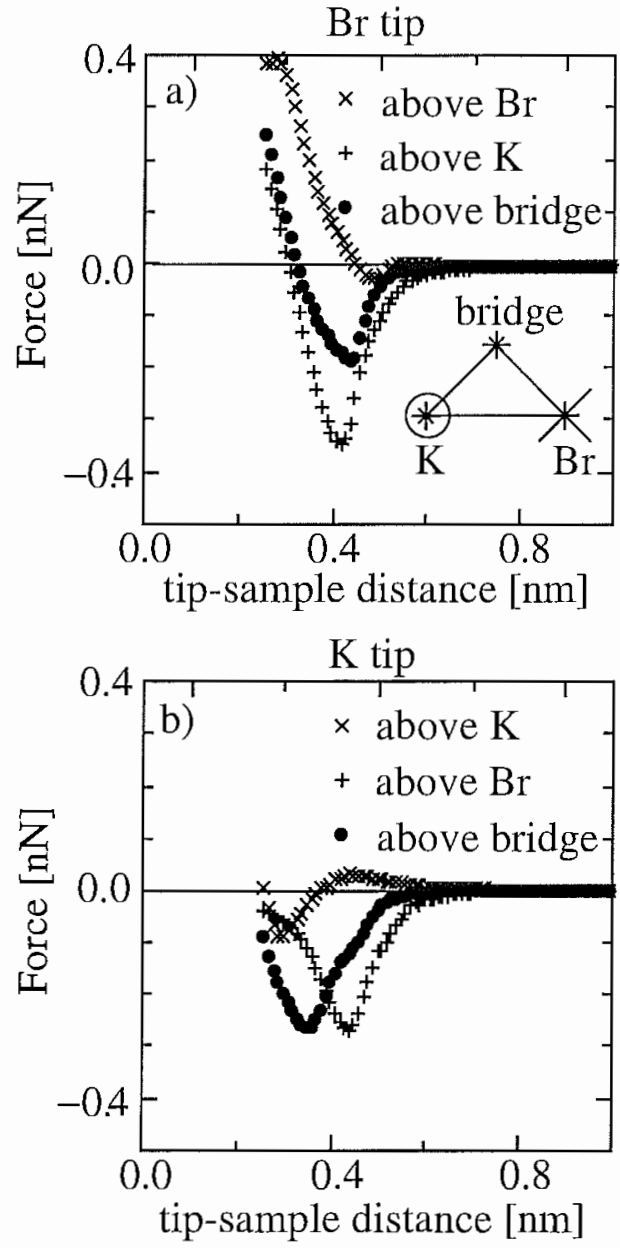

FIG. 1. Short-range force versus distance calculated for the (a) $\mathrm{Br}^{-}$and the (b) $\mathrm{K}^{+}$-terminated tip above a $\mathrm{K}^{+}$ion, above a $\mathrm{Br}^{-}$ion, and above the bridge position.

not at the same distance as the minimum obtained above the $\mathrm{Br}^{-}$ion: it is shifted about $0.1 \mathrm{~nm}$ closer to the sample. On the other hand, the $\mathrm{Br}^{-}$-terminated tip gives rise to minima of different magnitude but at about the same distance above the bridge position and the $\mathrm{K}^{+}$ion, Additional calculations for a given tip indicate that the force curves evolve continuously as the lateral position is changed between the three sites considered here. In the distance range studied, the results do not significantly change if the tip cluster is rotated with respect to its [111] axis perpendicular to the surface. Moreover, 110 atomic jumps occur, although tip-induced displacements of the surface ions affect the shape of the force curves [8], especially above the bridge site where a net electrostatic interaction with the tip apex ion solely arises as a result of relaxation. In particular, the two nearest $\mathrm{Br}^{-}$ions relax the most towards the tip when it is positively terminated. On the basis of these findings, a noncontact measurement of the force above the bridge position should allow one to 
distinguish between positively and negatively terminated tips. This idea inspired the following measurements.

Clean, flat $\mathrm{KBr}(001)$ surfaces were prepared by cleaving a single crystal in UHV and subsequently heating it at $100{ }^{\circ} \mathrm{C}$ for $1 \mathrm{~h}$. All measurements were carried out in a homebuilt UHV low-temperature SFM [15] at about $7 \mathrm{~K}$. We used a silicon cantilever with a spring constant $c_{L}$ of $40 \mathrm{~N} / \mathrm{m}$ and a free resonance frequency $f_{0}$ of $160622.47 \mathrm{~Hz}$. After introduction into ultrahigh vacuum, the cantilever was heated to $150{ }^{\circ} \mathrm{C}$ for $1 \mathrm{~h}$ without additional cleaning, e.g., by sputtering. We therefore believe that the tip was initially still covered with native silicon oxide. However, material was likely picked up from the sample, as suggested by numerous contrast changes in images recorded before an undistorted image of the surface lattice was obtained on a flat terrace. The SFM measurements described below were carried out on that terrace with the cantilever kept on resonance at a constant tip oscillation amplitude $A$ of $6.1 \mathrm{~nm}$, using excitation and frequency detection circuits controlled by a digital phaselocked loop [18]. Figure 2(a) shows a topographic image recorded at a constant frequency shift of $\Delta f=-19.5 \mathrm{~Hz}$, which corresponds to a normalized value $\gamma=$ $(\Delta f / f) c_{L} A^{3 / 2}[19]$ of $-2.31 \times 10^{-15} \mathrm{Nm}^{0.5}$. Similar images recorded after the frequency versus distance measurements discussed below, Figs. 2(b) and 2(c), showed the same undistorted contrast with a corrugation of $0.05 \mathrm{~nm}$.

We performed repeated measurements of the iuteraction-shifted resonance frequency as a function of the sample displacement above the four sites readily identified in undistorted images such as Figs. 2(a)-2(c)
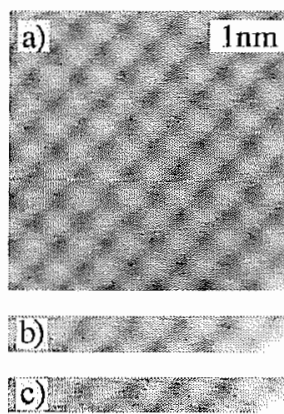

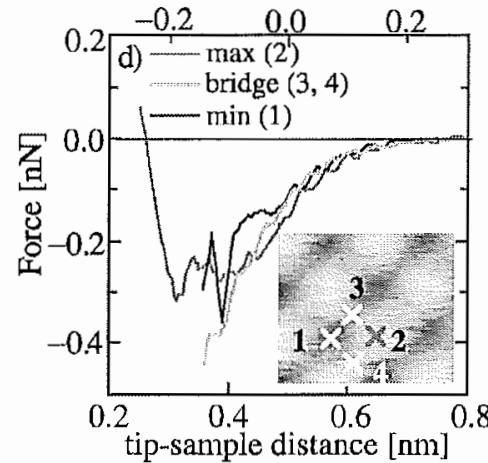

FIG. 2 (color online), Topographic images recorded at a constant frequency shift of $-19.5 \mathrm{~Hz}$ (a) before (b) between and (c) after all frequency versus distance measurements. Note that (b) and (c) cover the bottom part of (a) and show that the contrast of (a) is reproduced precisely. (d) Force versus distance data obtained from measurements above the maximum, above the minimum and above the bridge positions indicated in the inset. The horizontal scale is given by the sample displacement apart from an unknown offset. The origin of the top scale marks the mean location of the image in the inset. That of the bottom scale is the same as in Fig. 3 (see text). using a procedure similar to that described in Refs. [1214]. Special care was taken to minimize $z$-scale distortions caused by creep in the sample piezodrive. Differences between frequency shifts measured above the crystallographically equivalent bridge positions 3 and 4 can only be due to asymmetries of the tip. In the distance range analyzed here data measured above those positions coincided within experimental uncertainty. Thus the tip can be considered to be atomically sharp and symmetric, as assumed in our simulations. Forces were computed from the frequency shifts using a modified version of the numerical inversion method [20] briefly described in Ref. [21]. This procedure is meaningful as long as the frequency vs distance curves are nonhysteretic, i.e., exhibit no discontinuities. The data discussed further satisfy those conditions. Moreover, as already mentioned, images recorded at the same $\Delta f$ between frequency versus distance measurements revealed no significant changes.

We first attempted to separate the long-range van der Waals and electrostatic forces by subtracting an analytic fit to the long-range part [22] as clescribed in detail in an earlier publication on $\operatorname{KBr}(001)$ [13]; the nominal short-range forces obtained in this fashion are shown in Fig. 2(d). The distance dependence and magnitude of the force corresponding to site 2 compare reasonably well with the calculated ones; however, approximately comparable attractive forces are also obtained above sites 1 and 3 in contrast to our simulations. The reason could be that long-range forces are inadequately described by the fit at close tip-sample distances. In order to exclude any influence of residual siteindependent contributions we focus instead on differences of the forces obtained above the sites in question. The results are shown in Fig. 3.

The difference measured between sites 1 and 2 (top panel) follows the calculated dependence within a factor of about 1.75 for the $\mathrm{K}^{+}$-terminated tip and a factor of about 2 for the $\mathrm{Br}^{-}$-terminated tip. The experimental error is estimated to be about $30 \%$. Although the discrepancy between the measured and computed curves reflects limitations of our computational model, all three curves have similar shapes. The absolute distance to the surface could thus be determined within about $0.1 \mathrm{~nm}$ by aligning the experimental and calculated data such that the best agreement is found for the difference. The force difference obtained between sites 1 and 3 (middle panel) then also shows somewhat better agreement for the $\mathrm{K}^{+}$-terminated tip. However, the force difference between sites 3 and 2 (bottom panel) leaves no doubt that our measurements are best represented by a model assuming a positively terminated tip. Indeed, the force difference for the $\mathrm{K}^{+}$terminated tip changes sign at a distance of $0.4 \mathrm{~nm}$ in excellent agreement with the experimental curve, while in the case of the $\mathrm{Br}^{-}$-terminated tip this effect is absent. Therefore, we conclude that the electrostatic potential near the tip apex was positive in our experiment. This 

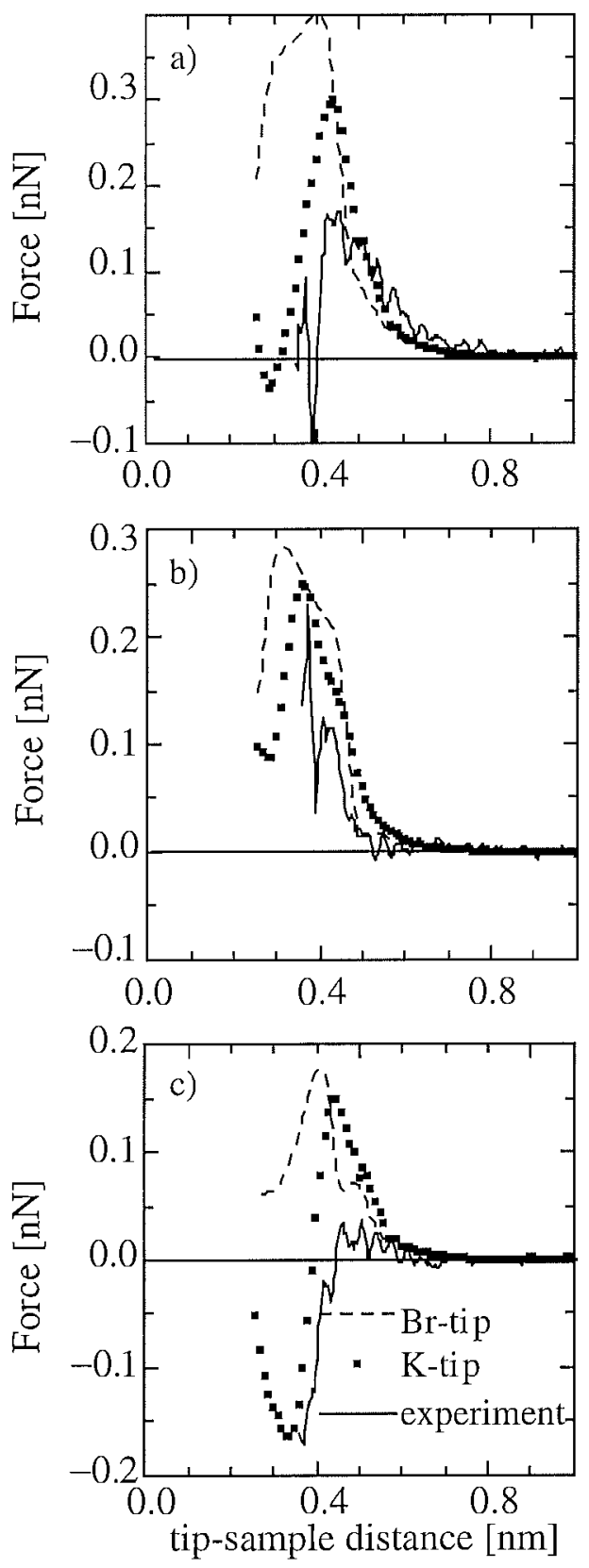

FIG. 3. Comparison of the calculated and the measured force differences for the force above (a) sites 1 and 2, (b) sites 1 and 3, (c) sites 3 and 2 identified in Fig. 2(d).

observation allows us to unequivocally identify the bright and dark spots in the images as $\mathrm{Br}^{-}$and $\mathrm{K}^{+}$ ions, respectively.

In summary, we have shown that a combination of atomistic simulations on an ionic crystal surface together with site-specific frequency versus distance measurements allows us to identify the location of surface species in noncontact SFM images even in such a difficult case as the $\mathrm{KBr}(001)$ surface. Site-independent forces can be eliminated by considering force differences between specific sites which can be readily identified in such images, provided that they are not distorted by tip asymmetries, noise, or drifts. This enables a direct comparison with short-range force-distance curves obtained from numerical simulations based on interaction potentials and tip apex models validated in previous studies. Our method should allow one to identify the charge and the location of surface point defects by performing force-distance measurements over judiciously chosen positions after first determining the sign of the electrostatic potential near the tip apex. The method demonstrated here will also prove useful for SFM experiments on other ionic systems where species recognition is difficult due to high symmetry of the surface.

This work was supported by the Swiss National Science Foundation and by its National Center of Competence in Research on Nanoscale Science. R. H. acknowledges funding from the Alexander von Humboldt Foundation during a postdoctoral year at McGill University, Montreal, Canada. The authors are grateful to M. A. Lantz, R. Bennewitz, and A. Shluger for useful discussions.

*Email address: r.hoffmann @ physik.uni-karlsr uhe.de

[1] F. J. Giessibl, Science 267, 68 (1995); S. Kitamura and M. Iwatsuki, Jpn. J. Appl. Phys. 34, L145 (1995).

[2] Noncontact Atomic Force Microscopy, edited by S. Morita, R. Wiesendanger, and E. Meyer (Springer, Berlin, 2002).

[3] A. Foster et al., in Ref. [2].

[4] M. Bammerlin et al., Probe Microscopy 1, 3 (1997).

[5] R. Bennewitz et al, Surf. Sci. Lett. 474, L197 (2001).

[6] R. Bennewitz et al., Phys. Rev. B 62, 2074 (2000).

[7] A.S. Foster, C. Barth, A. L. Shluger, and M. Reichling, Phys. Rev. Lett. 86, 2373 (2001); A. S. Foster et al., Phys. Rev. B 66, 235417 (2002).

[8] A. I. Livshits et al., Phys. Rev. B 59, 2436 (1999).

[9] P.V. Sushko, A. L. Shluger, and C. R. Catlow, Surf. Sci. 450, 153 (2000).

[10] G. Pacchioni, Surf. Rev. Lett. 7, 277 (2000).

[11] U. Heiz and W.-D. Schneider, J. Phys. D 33, R85 (2000).

[12] M. A. Lantz et al., Science 291, 2580 (2001).

[13] R. Hoffmann et al., Appl. Surf. Sci. 188, 238 (2002).

[14] R. Hoffmann et al., Phys. Rev. B 67, 085402 (2003).

[15] H. J. Hug et al, Rev. Sci. Instrum. 70, 3625 (1999).

[16] L. N. Kantorovich and A.S. Foster, SCIFI vl.0 Manual 1.3.2000.

[17] M. J. L. Sangster and R. M. Atwood, J. Phys. C 11, 1541 (1978)

[18] Ch. Loppacher et al., Appl. Phys. A 66, S215 (1998).

[19] F. J. Giessibl, Phys. Rev. B 56, 16010 (1997).

[20] F. J. Giessibl, Appl. Phys. Lett. 78, 123 (2001).

[21] O. Pfeiffer, et al., Phys. Rev. B 65, 161403(R) (2002).

[22] M. Guggisberg et al., Phys. Rev. B 61, 11151 (2000). 